\title{
Identification of the optimal cetuximab concentration that is effective against oral squamous cell carcinoma in collagen gel droplet embedded culture drug sensitivity testing
}

\author{
KANAME SAKUMA ${ }^{1}$, SHINTARO HANYU ${ }^{2}$, HARUKA TAKAHASHI ${ }^{2}$ and AKIRA TANAKA ${ }^{2}$ \\ ${ }^{1}$ Department of Oral and Maxillofacial Surgery, The Nippon Dental University, Niigata Hospital; \\ ${ }^{2}$ Department of Oral and Maxillofacial Surgery, The Nippon Dental University \\ School of Life Dentistry at Niigata, Niigata 951-8580, Japan
}

Received February 23, 2019; Accepted July 22, 2019

DOI: $10.3892 / \mathrm{mco} .2019 .1953$

\begin{abstract}
Anticancer drug sensitivity testing using the collagen gel droplet embedded culture drug sensitivity test (CD-DST) on oral squamous cell carcinoma (OSCC) samples beginning from 2010 has been conducted. The present study investigated the effect of adding cetuximab (Erbitux ${ }^{\circledR}$ ), a molecularly targeted drug, on anticancer drug activity against clinical OSCC specimens. A total of 25 specimens were obtained from 25 patients with OSCC between October 2013 and December 2017. The present study conducted anticancer drug sensitivity testing for cisplatin (CDDP), 5-fluorouracil (5-FU), cetuximab, three-drug combination, single agent and multi drug combinations, and cetuximab addition to the aforementioned regimens using CD-DST. In addition, the optimum concentration of each drug was evaluated. The overall evaluation success rate of the CD-DST method for OSCC specimens was 84.0\% (21 of 25 cases); sensitivity to anticancer drugs and cetuximab could be evaluated. The in vitro efficacy rate of a cetuximab single agent and CDDP $+5-\mathrm{FU}(\mathrm{PF})$ at a cut-off value of $50 \%$ was similar to the known clinical response rate. However, at a cut-off value of $50 \%$, the in vitro efficacy of PF + cetuximab was calculated to be $40 \%$, which was higher than the clinical response rate. The CD-DST method could be used to evaluate cetuximab, a molecularly targeted drug. Furthermore, its additive effect on conventional chemotherapy could be evaluated.
\end{abstract}

Correspondence to: Dr Kaname Sakuma, Department of Oral and Maxillofacial Surgery, The Nippon Dental University, Niigata Hospital, 1-8 Hamaura-cho, Chuo-ku, Niigata 951-8580, Japan

E-mail: sakuma.k@ngt.ndu.ac.jp

Abbreviations: ADCC, antibody-dependent cell-mediated cytotoxicity; CG, collagen gel-coated; EGFR, epidermal growth factor receptor; OSCC, oral squamous cell carcinoma; SCCHN, squamous cell carcinoma of the head and neck

Key words: anticancer drug sensitivity test, oral squamous cell carcinoma, collagen gel droplet embedded culture drug sensitivity test, cetuximab, anticancer drugs
The CD-DST method is suitable for evaluating and selecting chemotherapy regimens, including molecularly targeted drugs. Future studies are required to generate and evaluate relevant clinical data.

\section{Introduction}

Currently, there is no established methodology for the treatment of progressive, relapsed or metastatic in oral squamous cell carcinoma (OSCC). Cetuximab is a promising chemotherapeutic agent for treating OSCC that specifically binds to the epidermal growth factor receptor (EGFR). Cetuximab has been demonstrated to be associated with radiation therapy to improve local disease control rates and patient survival rates in locally advanced squamous cell carcinoma of the head and neck. In addition, treatment with cetuximab in conjunction with platinum drugs and fluorouracil was found to improve the survival of patients with recurrent or metastatic squamous cell carcinoma of head and neck (SCCHN) (1-4). However, there is no established predictor of treatment response in OSCC. Since the tumor response cannot be accurately predicted, patients are administered cetuximab regardless of tumor sensitivity. Moreover, cetuximab can result in infusion reactions in addition to other serious adverse events. Thus, the identification of predictors of treatment response in OSCC patients is essential to avoid ineffective drug administration and the risk associated with patients. Chemosensitivity tests used in the fields of colon cancer, stomach cancer and breast cancer are said to be effective for personalized medicine (5). In 1995, Kobayashi developed the CD-DST method, which is a chemical sensitivity test that combines a three-dimensional (3D) cell culture, serum-free culture, and an image-based colorimetric assay. The CD-DST method is used in many fields by overcoming many of the problems in chemical sensitivity tests that have been conducted so far $(5,6)$. Since CD-DST uses a micro-3D culture, it can be used to evaluate activity against OSCCs, as well as other types of cancer with a low tumor volume. Further, its use in personalized medicine initiatives to evaluate cytotoxic anticancer drug sensitivity of head and neck cancer has been described (7). In this study, we investigated whether the effectiveness of sensitivity testing in clinical specimens 
using cetuximab, a molecularly targeted drug, can be determined. The cut-off value of $\mathrm{T} / \mathrm{C} \%$ for assessing the efficacy of drug regimens including cisplatin (CDDP) and 5-fluorouracil (5-FU) in the presence or absence cetuximab in the CD-DST method was set to $40 \%$.

\section{Patients and methods}

Patients. Twenty-five OSCC patients who consented to participate in the study from October 2013 to December 2017 in Nippon Dental University at Oral and Maxillofacial Surgery, Niigata Hospital (Table I). The Ethics Committee of The Nippon Dental University School of Life Dentistry at Niigata (Niigaka, Japan) approved the present study (approval no. ECNG-H-119).

CD-DST was performed on 25 primary lesions and one metastatic lymph node; $5-7-\mathrm{mm}^{2}$ tissues (approximately $0.25-0.5 \mathrm{mg}$ ) were collected mainly from the area surrounding the hardened part of the tumor. CD-DST was performed in accordance with the methods described by Kobayashi et al $(6,8)$, using a human tumor cell primary culture system kit (Primaster ${ }^{\circledR}$; Kurabo Industries Ltd., Osaka, Japan). Briefly, the samples were treated with Dispersion Enzyme Cocktail EZ (Primaster ${ }^{\circledR}$ reagent). The samples comprised of cell suspensions were transferred into collagen gel-coated flasks (CG flasks, a Primaster ${ }^{\circledR}$ device) and incubated overnight in pre-culture medium, PCM-1 (Primaster ${ }^{\circledR}$ content). The collagen gel was digested with EZ to obtain viable cancer cells. Type I collagen, F-12 medium 10x concentrate, and reconstitution buffer were mixed in ice water at a 8:1:1 ratio (Primaster ${ }^{\circledR}$ content). The cancer cell suspension $\left(1 \times 10^{5}\right.$ cells $\left./ \mathrm{ml}\right)$ was then combined with the to the collagen solution. After $1 \mathrm{~h}$, DF medium supplemented with $10 \%$ fetal bovine serum was added to each well; the plates were placed in a $\mathrm{CO}_{2}$ incubator overnight at $37^{\circ} \mathrm{C}$. The following final concentrations of the anticancer drugs were added: $0.5 \mu \mathrm{g} / \mathrm{ml}$ cisplatin (CDDP; Randa Injection $50 \mathrm{mg} / 100 \mathrm{ml}$; Nippon Kayaku, Tokyo, Japan) (9); and $0.7 \mu \mathrm{g} / \mathrm{ml}$ fluorouracil (5-FU injection 250 Kyowa $^{\circledR}$; Kyowa Hakko, Tokyo, Japan) (9). The cancer cells were incubated for $24 \mathrm{~h}$. In addition, cetuximab (Erbitux ${ }^{\circledR}$ Injection $100 \mathrm{mg} / 20 \mathrm{ml}$; Merck Serono, Tokyo, Japan) was added at $250 \mu \mathrm{g} / \mathrm{dl}$ and incubated for $144 \mathrm{~h}$ as reported by Ryuki et al (10) (Table II). After removing the medium containing the 5-FU and CDDP anticancer agents, each well was washed twice with $3 \mathrm{ml}$ of Hanks' Balanced Salt Solution and covered with $4 \mathrm{ml}$ PCM-2 medium (Primaster ${ }^{\circledR}$ serum-free medium). When cetuximab was further added, it was incubated for 6 days after cetuximab addition. After the incubation, a final concentration of $50 \mu \mathrm{g} / \mathrm{ml}$ neutral red solution was added to each well. Surviving colonies in the collagen gel droplets were stained for $2 \mathrm{~h}$ and subsequently fixed in $10 \%$ neutral buffered formalin. After fixing, the samples rinsed with water and permitted to air-dry before being quantified by an optical density image analysis using Primage System ${ }^{\circledR}$ (Solution Systems, Tokyo, Japan) (Fig. 1). In vitro sensitivity was expressed as the $\mathrm{T} / \mathrm{C}$ ratio of the optical density, where $\mathrm{T}$ represents the treated samples and $\mathrm{C}$ represents the controls; a $\mathrm{T} / \mathrm{C}$ ratio of $<50 \%$ was regarded as in vitro chemosensitivity. A tumor cell colony volume ratio (tumor growth rate) at 0 time was calculated using the control
Table I. Patient characteristics $(n=25)$.

Characteristics

No. of patients $(\%)$

Sex

Male

$15(60.0)$

Female

$10(40.0)$

Age, mean, range

$70.6,37-93$

Histology (Squamous cell carcinoma)

Differentiation

$19(76.0)$

Moderately differentiated

$5(20.0)$

Poorly differentiated

$1(4.0)$

Stage

I

$3(1.2)$

II

$9(36.0)$

3 (12.0)

III

$10(40.0)$

Primary site

Tongue

9 (36.0)

Buccal mucosa

6 (24.0)

Oral floor

2 (8.0)

Gingival

$6(24.0)$

Hard palate

2 (8.0)

Resection mode

Biopsy

$20(80.0)$

Surgery

5 (20.0)

Sample site

Neck metastasis

Primary

$24(96.0)$

CD-DST

Success

$21(84.0)$

Failure

4 (16.0)

CD-DST, collagen gel droplet-embedded culture drug sensitivity test.

Table II. Anticancer agents and concentrations used in the CD-DST.

\begin{tabular}{lcc}
\hline Agents & Concentration $(\mu \mathrm{g} / \mathrm{ml})$ & Time $(\mathrm{h})$ \\
\hline CDDP & 0.5 & 24 \\
5 -FU & 0.8 & 24 \\
Cetuximab & 250 & 144 \\
\hline
\end{tabular}

CD-DST, collagen gel droplet-embedded culture drug sensitivity test.

group; a value of less than 0.8 was regarded as unsuccessful culture (low growth rate), regardless of the tumor cell colony volume in the control group.

Statistical analysis. Fisher's exact test was used to determine differences between groups. A value of $\mathrm{P}<0.05$ was considered statistically significant (Ekuseru-Toukei 2015, Social Survey Research Information Co., Ltd.). 


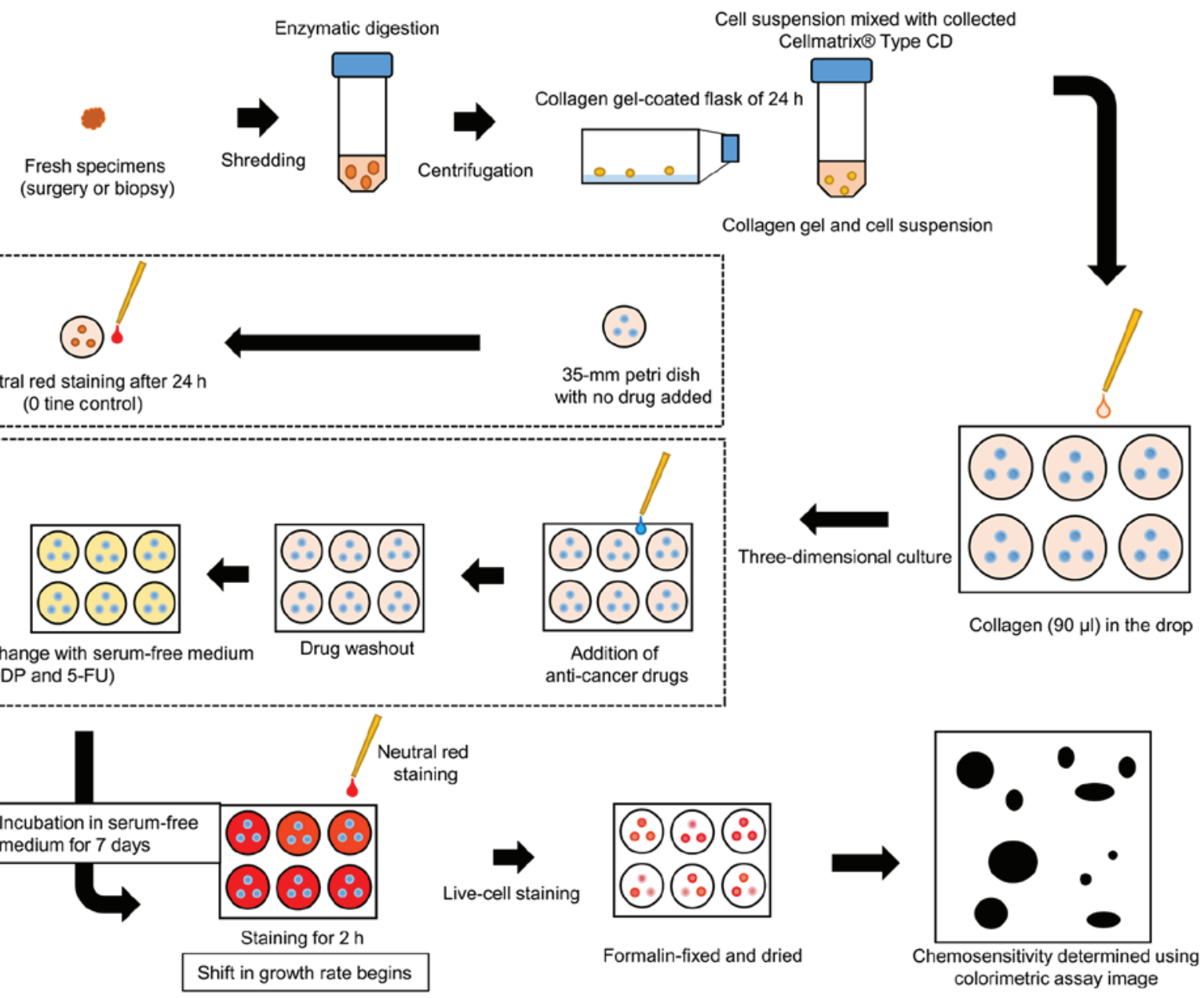

Figure 1. Overview of the collagen gel droplet embedded culture drug sensitivity test method. CDDP, cisplatin; 5-FU, 5-fluorouracil.

\section{Results}

Evaluation success rate using clinical specimens. CD-DST of cetuximab against OSCC had an overall evaluation success rate of $84.0 \%$ ( 21 of 25 cases), and could be applied to all anticancer agents used in the present study. The evaluation success rate was $83.3 \%$ ( 20 of 24 cases) for primary lesions and $100 \%$ ( 1 of 1 case) for metastatic lymph nodes (Table III). The causes of unsuccessful CD-DST were insufficient tumor cells (growth rate less than 0.8) in 2 cases and bacterial contamination in 2 cases. The mean tumor growth rate was $6.13 \pm 4.82$. In addition, no significant difference was observed between sensitivity to various anticancer agents, stage, or histological differentiation (n.s.).

Comparison between in vitro efficacy rate and clinical response rate to various anticancer drugs. We compared the in vitro efficacy rate and clinical response rate of representative anticancer drugs used for clinical treatment of OSCC $(2,11)$ (Table IV). PF's in vitro efficacy rate was $23.8 \%$, which was similar to its clinical response rate of $20-30 \%(2,11)$. In addition, the in vitro efficacy rate of single agent cetuximab was $11.1 \%$, which was similar to its clinical response rate of $13 \%$. However, $\mathrm{PF}+$ cetuximab had an in vitro efficacy rate of $52.0 \%$ versus a clinical response rate of $36.0 \%$ (2).
Determination of cut-off value of PF + cetuximab in CD-DST. In anticancer drug sensitivity testing, conducted using the CD-DST method in the present study, the in vitro efficacy rate of PF + cetuximab was $52.3 \%$ at a cut-off value of $50 \%$, which was higher than the clinical response rate. The cut-off value calculated with CD-DST data using PF + cetuximab in 21 samples and the clinical response rate (2) of PF + cetuximab, the cut-off value was $40 \%$, which was similar to the clinical response rate of $33.3 \%$ (Fig. 2).

\section{Discussion}

Recently, the molecular targeting drug cetuximab has been used in combination with conventional anticancer agents for relapsed and metastatic SCCHN $(12,13)$. Cetuximab competitively binds to EGFR expressed on the tumor and shows an antitumor effect by suppressing signal transduction $(14,15)$. Specific biomarkers in anti-tumor effects have resulted in elimination of ineffective agents for chemotherapy, development of personalized chemotherapy, improvement of treatment response, and improvement of patients' QOL. However, biomarkers for cetuximab in the OSCC have not been defined. As a result, personalized therapy for molecular targeted therapies was not currently available to patients with OSCC. Therefore, predicting the therapeutic response of cetuximab in OSCC patients can avoid ineffective drug 
Table III. Evaluation of the success rate of the CD-DST using oral squamous cell carcinoma clinical specimens.

\begin{tabular}{lccc}
\hline Sample site & Number of samples & Evaluable cases & Evaluation success rate, \% \\
\hline Primary lesion & 24 & 20 & $83.3(20 / 24)^{\mathrm{a}}$ \\
Metastatic lymph node & 1 & 1 & $100(1 / 1)^{\mathrm{a}}$ \\
Total & 25 & 21 & $84.0(21 / 25)^{\mathrm{a}}$ \\
\hline
\end{tabular}

${ }^{a}$ Number of success cases/total number of assays. CD-DST, collagen gel droplet-embedded culture drug sensitivity test.

Table IV. In vitro sensitivity and clinical efficacy rates of individual drugs against oral squamous cell carcinoma.

\begin{tabular}{lccc}
\hline Efficacy rate & PF & Cetuximab, 250 $\mu \mathrm{g}$ & PF + Cetuximab \\
\hline CD-DST in vitro efficacy rate, $\%$ & $23.8(5 / 21)^{\mathrm{a}}$ & $11.1(2 / 18)^{\mathrm{a}}$ & $52.3(11 / 21)^{\mathrm{a}}$ \\
Clinical efficacy rate, $\%$ & $20.0-30.0(2,11)$ & $13.0(2)$ & $36.0(2)$
\end{tabular}

${ }^{a}$ Number of effective cases/total number of assays. In vitro drug sensitivity was defined as positive when the T/C ratio was $\leq 50 \%$. PF, cisplatin + 5-fluorouracil; CD-DST, collagen gel droplet-embedded culture drug sensitivity test.

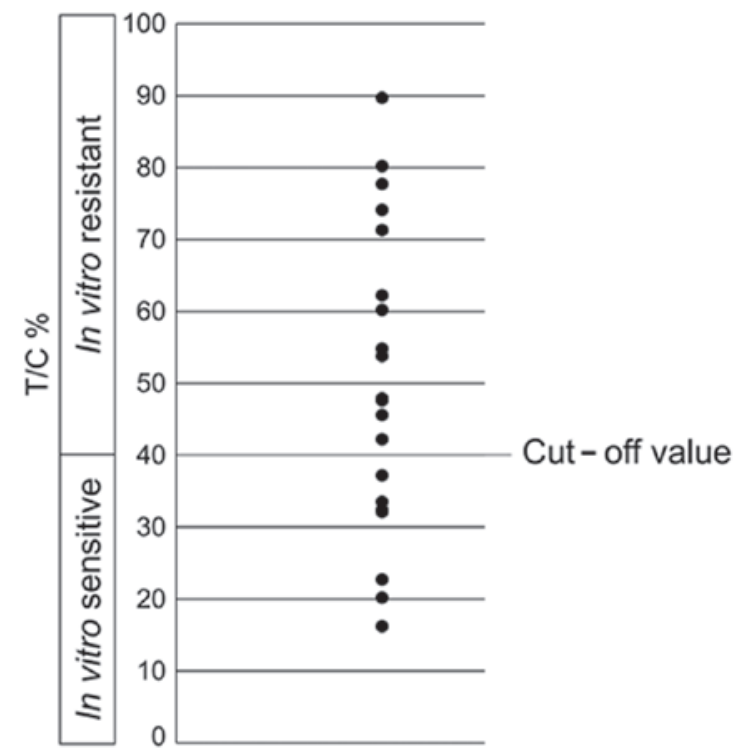

Figure 2. Determination of cut-off value of CDDP+5-FU+Cetuximab in CD-DST. In total, 21 cases who performed the CD-DST method with anticancer drug contact of CDDP 5-FU+Cetuximab are arranged in order of $\mathrm{T} / \mathrm{C} \%$. The sensitivity of CD-DST for CDDP+5-FU+Cetuximab in oral cancer was found to be high when $\mathrm{T} / \mathrm{C} \%<40 \%$, and lower when it was $>40 \%$. CD-DST, collagen gel droplet-embedded culture drug sensitivity test; CDDP, cisplatin; 5-FU, 5-fluorouracil; $\mathrm{PF}, \mathrm{CDDP}+5-\mathrm{FU}$; T/C, total colony of the treated cells/total colony of the untreated cells.

administration and can avoid the patient adverse events associated therewith.

The CD-DST we performed in the present study has a potentially high success rate, based on initial culture, needs a small number of cells, and uses serum-free culture medium and image colorimetry to eliminate the influence of fibroblasts. The CD-DST has the advantage of using an anticancer drug concentration comparable to the clinical therapeutic dose $(5,6,9,10)$.
In fact, the primary measurement success rate of the CD-DST was reported to be $87.5 \%$ in colorectal cancer, $79.2 \%$ in lung cancer, and $84.3 \%$ in breast cancer (16-18), and it shows a high clinical efficacy prediction rate of $91 \%$ (5). Similarly, in sensitivity testing using cisplatin, fluorouracil, or docetaxel in OSCC, as we reported earlier, the primary culture success rate was $81.8 \%$ (19). In the present study, we conducted anticancer drug sensitivity testing, including cetuximab specimens of OSCC cases, with a success rate of $83.3 \%$ (20 of 25 cases) in primary tumors, $100 \%$ (1 out of 1 case) in metastatic lymph nodes, and $84.0 \%$ overall ( 21 of 25 cases). This result suggested that the CD-DST method could also evaluate anticancer drugs including cetuximab in OSCC.

Kobayashi et al $(5,6)$ reported a statistically significant correlation between in vitro efficacy in CD-DST and clinical response of breast cancer, gastric cancer, colorectal cancer, and lung cancer. In the present study on OSCC, the $23.8 \%$ in vitro efficacy rate of PF therapy was comparable to the previously reported 20.0-30.0\% clinical response rate to preoperative $\mathrm{PF}$ chemotherapy $(2,11)$. Further, the $11.1 \%$ in vitro efficacy rate of cetuximab therapy in the present study was comparable to the reported clinical response rate of $13.0 \%$ (2). Ryuki et al (10) reported a concentration of cetuximab alone in the CD-DST method using a human oral cancer cell line of $250 \mu \mathrm{g} / \mathrm{ml}$. We used cetuximab alone at $250 \mu \mathrm{g} / \mathrm{ml}$ based on the above study. We found the in vitro efficacy was consistent with the clinical response rate. However, when the CD-DST method was performed with $\mathrm{PF}+$ cetuximab $(250 \mu \mathrm{g} / \mathrm{ml})$, the in vitro response rate was $52.3 \%$, which was higher than the clinical response rate of $36.0 \%$. The in vitro efficacy rate after changing the cut-off value from 50 to $40 \%$ was $33.3 \%$ ( 7 out of 21 ), similar to the clinical response rate of $36.0 \%$. The sensitivity of CD-DST for PF + cetuximab in oral cancer was found to be high when $\mathrm{T} / \mathrm{C} \%$ was less than $40 \%$, and lower when it was more than $40 \%$.

We previously performed CD-DST in a recurrent postoperative course of hard palate cancer (T2N2 cM0: Stage IVA) 
and compared the results to those of sensitivity testing of $\mathrm{PF}+$ cetuximab and its clinical efficacy (20). In the same study, the $\mathrm{T} / \mathrm{C} \%$ of the CD-DST method was found to represent high sensitivity at $32.4 \%$, and the clinical efficacy of $\mathrm{PF}+$ cetuximab was consistent with the partial response (20). The reported $\mathrm{T} / \mathrm{C} \%$ is $32.4 \%$, which is less than $40 \%$ of the cut-off value we determined in the present study, and can be considered to represent high sensitivity. Further, because it is consistent with clinical efficacy, the cut-off value of $40 \%$ determined in the present study appears appropriate. In addition, the antitumor effects of cetuximab include antibody-dependent cell-mediated cytotoxicity (ADCC) activity in addition to its inhibitory effect on EGFR signaling (21). Although it is challenging to evaluate ADCC activity in vitro, a comprehensive evaluation was possible in the present study because the drug effect was assessed based on results of CD-DST and clinical response rate.

The mechanism by which the antitumor effect is enhanced has not been demonstrated when cetuximab is used in combination with conventional anticancer agents. It has been suggested that the CD-DST method can contribute to the development of individualized chemotherapy by predicting the treatment effect when the anticancer drug is administered with cetuximab in the upper part. Cetuximab alone and the effect of adding cetuximab to combination therapy can be evaluated.

Chemotherapy, including molecularly targeted drugs, can increase the physical and economic burden on patients and affect their quality of life. Therefore, it was suggested that the application of CD-DST method could become an increasingly important test method in the future.

\section{Acknowledgements}

Not applicable.

\section{Funding}

No funding was received.

\section{Availability of data and materials}

The datasets used and/or analyzed during the current study are available from the corresponding author on reasonable request.

\section{Authors' contributions}

KS and SH have made substantial contributions to the conception and design of the study, and the acquisition, analysis and interpretation of data. HT and AT were involved in drafting the manuscript and critically revised it for important intellectual content. HT and AT made substantial contributions to the conception and design of the study, and the acquisition, analysis and interpretation of data.

\section{Ethics approval and consent to participate}

The patients consented to participate in the study. The Ethics Committee of The Nippon Dental University School of Life Dentistry at Niigata (Niigata, China) approved the present study (approval no. ECNG-H-119).

\section{Patient consent for publication}

Written informed consent was obtained from the patients.

\section{Competing interests}

The authors declare that they have no competing interests.

\section{References}

1. Bonner JA, Harari PM, Giralt J, Azarnia N, Shin DM, Cohen RB, Jones CU, Sur R, Raben D, Jassem J, et al: Radiotherapy plus cetuximab for squamous-cell carcinoma of the head and neck. N Engl J Med 354: 567-578, 2006

2. Vermorken JB, Mesia R, Rivera F, Remenar E, Kawecki A, Rottey S, Erfan J, Zabolotnyy D, Kienzer HR, Cupissol D, et al: Platinum-based chemotherapy plus cetuximab in head and neck cancer. N Engl J Med 359: 1116-1127, 2008.

3. Rivera F, García-Castaño A, Vega N, Vega-Villegas ME and Gutiérrez-Sanz L: Cetuximab in metastatic or recurrent head and neck cancer: The EXTREME trial. Expert Rev Anticancer Ther 9: 1421-1428, 2009.

4. Yoshino T, Hasegawa Y, Takahashi S, Monden N, Homma A, Okami K, Onozawa Y, Fujii M, Taguchi T, de Blas B, et al: Platinum-based chemotherapy plus cetuximab for the first-line treatment of Japanese patients with recurrent and/or metastatic squamous cell carcinoma of the head and neck: Results of a phase II trial. Jpn J Clin Oncol 43: 524-531, 2013.

5. Kobayashi H, Tanisaka K, Doi O, Kodama K, Higashiyama M, Nakagawa H, Miyake M, Taki T, Hara S, Yasutomi M, et al: An in vitro chemosensitivity test for solid human tumors using collagen gel droplet embedded cultures. Int J Oncol 11: 449-455, 1997.

6. Kobayashi H: Development of a new in vitro chemosensitivity test using collagen gel droplet embedded culture and image analysis for clinical usefulness. Recent Results Cancer Res 161: 48-61, 2003.

7. Shintani S, Hino S, Nakashiro K and Hamakawa H: Clinical trial of chemotherapy identified according to chemosensitivity assay for oral cancer patients with unresectable recurrent lesions. Gan To Kagaku Ryoho 33: 357-360, 2006 (In Japanese)

8. Kobayashi H, Tanisaka K, Kondo N, Mito Y, Koezuka M, Yokouchi H, Higashiyama M, Kodama K, Doi O and Yamada M: Development of new in vitro chemosensitivity test using collagen gel droplet embedded culture and its clinical usefulness. Gan To Kagaku Ryoho 22: 1933-1939, 1995 (In Japanese).

9. Sakuma K, Tanaka A and Mataga I: Collagen gel dropletembedded culture drug sensitivity testing in squamous cell carcinoma cell lines derived from human oral cancers: Optimal contact concentrations of cisplatin and fluorouracil. Oncol Lett 12: 4643-4650, 2016.

10. Ryuki T, Sakuma K and Tanaka A: Optimal cetuximab contact concentration using a collagen gel droplet-embedded culture drug chemosensitivity test in human oral squamous carcinoma cell lines. Chemotherapy 7: 253-260, 2018.

11. Kiyota N, Tahara M, Kadowaki S, Fuse N, Doi T, Minami H and Ohtsu A: Systemic chemotherapy with cisplatin plus 5-FU (PF) for recurrent or metastatic squamous cell carcinoma of the head and neck (R/M SCCHN): Efficacy and safety of a lower dose of PF (80/800) at a single institution in Japan. Jpn J Clin Oncol 39: 225-230, 2009.

12. Saijo N: Molecular target therapy in cancer. Nihon Rinsho 68 1779-1786, 2010 (In Japanese).

13. Saijo N: Molecular targeted therapy for lung cancer, recent topics. J Lung Cancer 7: 1-8, 2008.

14. Lurje $G$ and Lenz HJ: EGFR signaling and drug discovery. Oncology 77: 400-410, 2009.

15. Huang SM, Bock JM and Harari PM: Epidermal growth factor receptor blockade with $\mathrm{C} 225$ modulates proliferation, apoptosis, and radiosensitivity in squamous cell carcinomas of the head and neck. Cancer Res 59: 1935-1940, 1999.

16. Araki Y, Isomoto H, Matsumoto A, Kaibara A, Yasunaga M, Hayashi K, Yatsugi $\mathrm{H}$ and Yamauchi K: An in vitro chemosensitivity test for colorectal cancer using collagen-gel droplet embedded cultures. Kurume Med J 46: 163-166, 1999.

17. Kawamura $M$, Inoue $Y$, Oyama $T$ and Kobayashi $H$ : Chemosensitivity test for unresectable non-small cell lung cancer. Nihon Geka Gakkai Zasshi 103: 229-232, 2002 (In Japanese). 
18. Takamura Y, Kobayashi H, Taguchi T, Motomura K, Inaji H and Noguchi S: Prediction of chemotherapeutic response by collagen gel droplet embedded culture-drug sensitivity test in human breast cancers. Int J Cancer 98: 450-455, 2002.

19. Sakuma K, Tamura R, Hanyu S, Takahashi H, Sato H, Oneyama T, Yamaguchi A and Tanaka A: Clinical study on collagen gel droplet-embedded culture drug sensitivity test for multidrug combination chemotherapy and super selective intraarterial infusion chemoradiotherapy in oral squamous cell carcinoma. Mol Clin Oncol 7: 1021-1026, 2017.
20. Sakuma K, Tamura R, Noda N, Mizutani M, Yamaguchi A and Tanaka A: Collagen gel droplet-embedded culture drug sensitivity testing in hard palate cancer-predicted antitumor efficacy of cetuximab: A case report. Mol Clin Oncol 7: 637-641, 2017.

21. Kimura H, Sakai K, Arao T, Shimoyama T, Tamura T and Nishio K: Antibody-dependent cellular cytotoxicity of cetuximab against tumor cells with wild-type or mutant epidermal growth factor receptor. Cancer Sci 98: 1275-1280, 2007. 